\title{
КЛИНИКО-ПРОГНОСТИЧЕСКИЙ АНАЛИЗ ЭКСПРЕССИИ PD-L1 И COX-2
}

\section{CLINICAL AND PROGNOSTIC ANALYSIS OF THE EXPRESSION OF PD-L1 AND COX-2}

\section{Soltanova}

Summary. In modern oncology, the determination of PD-L1and COX-2 in the tumor tissue is one of the promising areas of research that allow you to correctly and adequately make a plan for further treatment of the patient and assess the prognosis of the disease. Determination of the expression level of the PDL-1 molecule and COX-2 is considered as a potential biomarker for predicting the effectiveness and duration of treatment of malignant neoplasms. The lesion of the lymph nodes in cervical cancer is one of the main risk factors for the dissemination of the disease. Metastases in the pelvic lymph nodes are associated with a decrease in the 5 -year survival rate of patients from $85 \%$ to $53 \%$. From this position, due to the expansion of the opportunities of adjuvant therapy in this category of patients, the study of the receptor profile (PD-L1, COX-2) of tumor cells is of particular relevance. Interpretation of the results of immunohistochemical staining of cervical cancer with antibodies to PD-L1 and COX-2 in a cohort of patients with lymph node metastases (N1) revealed the presence of certain regularities between the expression of PD-L1 and COX-2 and clinical prognostic parameters.

Keywords: cervical cancer, programmed death, ligand, cyclooxygenase, lymph nodes, oncology.

\section{Ввемение}

B современной онкологии определение PD-L1 и COX-2 в опухолевой ткани является одним из перспективных направлений исследований, позволяющих правильно и адекватно составить план дальнейшего лечения пациента и оценить прогноз заболевания. Определение уровня экспрессии молекулы PDL-1 и ЦОГ-2 рассматривается как потенциальный биомаркер для прогнозирования эффективности и продолжительности лечения злокачественных новообразований.

Наличие метастатических лимфатических узлов при раке шейки матки является одним из неблагоприятных прогностических факторов, существенно влияющим на показатели выживаемости больных. Так, по данным
Солтанова Ирада Фахраддин кызы

Докторант, Азербайджанский медицинский университет, г. Баку, Азербайджан

geray.80@mail.ru

Аннотация. B современной онкологии определение PD-L1 и COX-2 в опухолевой ткани является одним из перспективных направлений исследований, позволяющих правильно и адекватно составить план дальнейшего лечения пациента и оценить прогноз заболевания. Определение уровня экспрессии молекулы PDL-1 и COX-2 рассматривается как потенциальный биомаркер для прогнозирования эффективности и продолжительности лечения злокачественных новообразований. Поражение лимфатических узлов при раке шейки матки - один из основных факторов риска распространения болезни. Метастазы в тазовые лимфатические узлы связаны со снижением 5-летней выживаемости пациентов с 85\% до 53\%. С этой позиции в связи с расширением возможностей адъювантной терапии у данной категории пациентов изучение рецепторного профиля (PD-L1, (OХ-2) опухолевых клеток приобретает особую актуальность. Интерпретация результатов иммуногистохимического окрашивания рака шейки матки антителами к PD-L1 и COX-2 в когорте пациентов с метастазами в лимфатические узлы (N1) выявила наличие определенных закономерностей между экспрессией PD-L1 и COX-2 и клинические прогностические параметры.

Ключевые слова: рак шейки матки, лиганд, запрограммированная смерть, циклооксигеназа, лимфатические узлы, онкология.

Y. Chen et al., метастазы в тазовых лимфатических узлах ассоциируются со снижением 5-летней выживаемости больных с 85 до 53\% [3]. Опухолевое поражение регионарных узлов при РШМ считается индикатором риска отдалённого метастазирования [4, с.12], в связи с чем данная категория больных нуждается в адьювантной терапии, специфика и объём которой варьируют в зависимости от множества факторов [2]. С другой стороны, распространённое в последнее время исследование биологических маркёров опухолей шейки матки (PD-L1, COX-2) также направлено на оптимизацию режимов системной терапии [8]. Поэтому изучение корреляционных отношений между метастазированием в лимфатические узлы и наличием в опухоли рецепторов PD-L1 [6] и COX-2 позволит более целенаправленно подходить к выбору больных, нуждающихся в адьювантном лечении. 
Таблица 1. Зависимость уровня экспрессии СОХ-2-рецепторов при РШМ от стадии заболевания

\begin{tabular}{|c|c|c|c|c|c|c|c|c|c|}
\hline \multirow{2}{*}{$\begin{array}{l}\text { Уровень } \\
\text { СОХ-2 } \\
\text { экспрессии }\end{array}$} & \multicolumn{2}{|c|}{ IA } & \multicolumn{2}{|l|}{ IB } & \multicolumn{2}{|c|}{ IIA } & \multicolumn{2}{|c|}{ IIIC1 } & Всего \\
\hline & \multicolumn{9}{|c|}{ n / (\%) } \\
\hline $1+$ & 5 & $71.4 \pm 17.1$ & 10 & $20.4 \pm 5.8$ & 1 & $25.0 \pm 21.7$ & 1 & $10.0 \pm 9.5$ & \multirow{3}{*}{$\begin{array}{l}P \times 2=0.01 \\
P H=0.224\end{array}$} \\
\hline $2+$ & 0 & 0 & 21 & $42.9 \pm 7.1$ & 0 & 0 & 7 & $70.0 \pm 14.5$ & \\
\hline $3+$ & 2 & $28.6 \pm 17.1$ & 18 & $36.7 \pm 6.9$ & 3 & $75.0 \pm 21.7$ & 2 & $20.0 \pm 12.6$ & \\
\hline
\end{tabular}

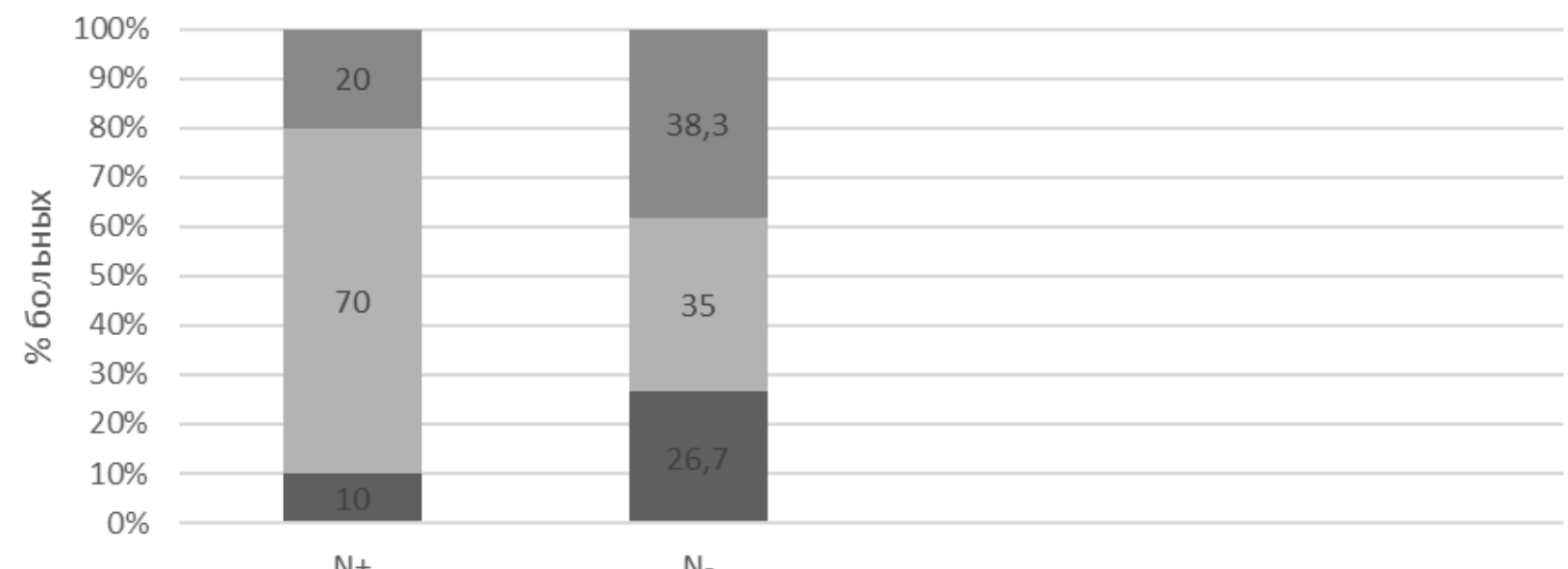

$\mathrm{N}+$

$\mathrm{N}-$

- $\operatorname{coX}-21+\quad \mathrm{COX}-2 \mathrm{a}+\mathrm{COX}-23+$

$\mathrm{PX}=0,110 ; \mathrm{PH}=0,865$

Рис. 1. Соотношение COX-2-экспрессирующих опухолей (в \%) у больных с метастазами в лимфатические узлы

\section{Шель исслеАования}

Исследование зависимости между экспрессией PDL1 и COX-2 в опухолевых клетках при РШМ и частотой метастазирования в регионарные лимфатические узлы.

\section{Материалы и методы}

В исследование включено 70 женщин с гистологически подтверждённым диагнозом первичного РШM I-IIA и IIIC1 стадий, получивших хирургическое лечение в Онкологической Клинике АМУ за период с 2015 по 2019 гг. Наряду со стандартным морфологическим исследованием, был использован стандартный режим иммунногистохимической (ИГХ) обработки (VENTANA Bench Mark Ultra) с помощью кроличьих моноклональных антител к COX-2 (SP21) и PD-L1 (VD21R) фирмы Medaysis [1, с. 11]. Уровень экспрессии PD-L1 оценивался по количеству тропных к антителам клеток - при окрашивании менее $1,0 \%$ от общей попу- ляции результат считался негативным [10, с. 265], при окрашивании более 1,0\% - позитивным [5, с. 66]. По уровню экспрессии COX-2 в плоском эпителии были выделены четыре группы с цитоплазматическим окрашиванием: 0 (негативная реакция), 1+ (слабая степень экспрессии), 2+ (средняя) и 3+ (выраженная). Статистическая обработка полученных результатов осуществлялась с применением дискриминантного - $\mathrm{x}$ 2-Pearson и непараметрического вариационного анализа H-Kruskal-Wallis на базе программы SPSS-26. Разница считалась достоверной при доверительной вероятности не менее $95 \%$ (уровень $\mathrm{p}<0,05$ ).

\section{Результаты исслеАования}

Возраст больных колебался в пределах 32-71 год,


прошли стандартный объём обследований, предусмотренный для РШМ: клинический осмотр, лабораторные и инструментальные методы (УЗИ, МРТ и др.). В контек- 
Таблица 2. Зависимость уровня экспрессии PD-L1 -рецепторов при PШM от стадии заболевания

\begin{tabular}{l|l|l|l|l|l|l|l|l|l|}
\multirow{2}{*}{$\begin{array}{l}\text { PD-L1 } \\
\text { экспрессия }\end{array}$} & IA & IB & IIA & IIIC1 & Bсего \\
\cline { 2 - 10 } & n /\% & 100 & 38 & $77.6 \pm 6.0$ & 4 & 100 & 2 & $20.0 \pm 12.6$ \\
\hline PD-L1-negative & 7 & 0 & 11 & $22.4 \pm 6.0$ & 0 & 0 & 8 & $80.0 \pm 12.6$ \\
\hline PD-L1-positive & 0 & &
\end{tabular}



Рис. 2. Соотношение PD-L1-негативных и PD-L1-позитивных вариантов опухоли (в \%) у больных с метастазами в лимфатические узлы

сте проводимой работы ценность представляют заключения МРТ в качестве источника получения информации о состоянии регионарных лимфатических узлов на предоперационном этапе. По данным МРТ, увеличенные лимфатические узлы (размером до 14 мм) были обнаружены у 4-х больных, из них в 2-х случаях это были пресакральные и в 2-х - лимфатические узлы мезоректума. Всем больным была произведена экстирпация матки с придатками в различных вариантах, в зависимости от степени распространённости опухолевого процесса. Распределение по стадиям было следующим:

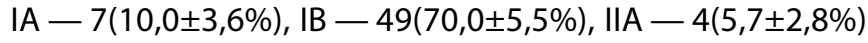

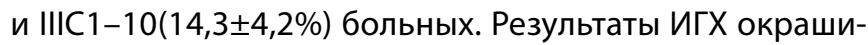
вания антителами к COX-2-рецепторам представлены в таблице 1. В целом частота встречаемости COX-2-экспрессирующих опухолей выраженностью 1+, 2+ и 3+

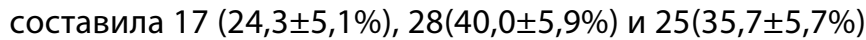
случаев соответственно. Как видно из приведённых данных, экспрессия СОХ-2 уровня 1+, 2+ и 3+ в ІА стадии составила $5(71,4 \pm 17,1 \%), 0$ и $2(28,6 \pm 17,1 \%)$ случая соответственно. В ІВ стадии эти показатели были равны $10(20,4 \pm 5,8 \%), 21(42,9 \pm 7,1 \%)$ и $18(36,7 \pm 6,9 \%)$ соответственно. Во ІІА стадии число больных с СОХ-2 1+,

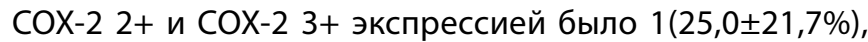

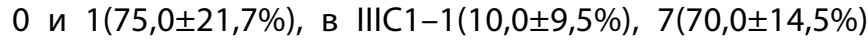


зультаты свидетельствуют об отсутствии достоверной корреляции (не подтверждённой по Kruskal-Wallis) между выраженностью СОХ-2-статуса и стадией.

На рисунке 1 приведена зависимость между степенью экспрессии СОХ-2 и метастазированием в лимфатические узлы. В группе больных без метастазов в лимфатические узлы (N0) частота встречаемости СОХ-2 1+, 2+ и 3+ вариантов РШМ составила $16(26,7 \pm 5,7 \%), 21(35,0 \pm 6,2 \%)$ и $23(38,3 \pm 6,3 \%)$ случая соответственно.

В группе больных с метастазами в лимфатических узлах (N1) распределение было следующим: 1(10,0土9,5\%), 
$7(70,0 \pm 14,5 \%)$ и $2(20,0 \pm 12,6 \%), P=0,110, P H=0,865$. Как показывают результаты, по данной категории достоверной корреляции не прослеживается, следовательно, наличие и выраженность СОХ-2 экспрессии не влияет на частоту регионарного метастазирования.

В таблице 2 отражены результаты ИГХ исследования PD-L1-рецепторов при РШМ в зависимости от стадии заболевания. Согласно приведённым данным, в ІА стадии PD-L1-позитивных опухолей не наблюдалось, в IB стадии соотношение PD-L1+ и PD-L1- форм PШМ было

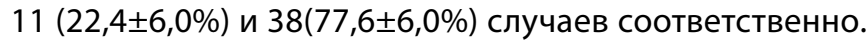
Bo IIA стадии все опухоли были PD-L1-негативны. B IIIC1 стадии PD-L1 экспрессия отсутствовала в 2(20,0 $12,6 \%)$


зитивны $(\mathrm{P}<0,001, \mathrm{PH}<0,001)$.

Из этого следует, что PD-L1-экспрессия достоверно ассоциируется с IIIC1 стадией заболевания, характеризующейся наличием метастазов в тазовых лимфатических узлах.

На рис. 2 отражена диаграмма распределения PDL1+ и PD-L1- опухолей среди больных с метастазами в лимфатические узлы. Как представлено на диаграмме, в группе пациентов с отсутствием метастазов в тазовых лимфатических узлах (N0) соотношение PD-L1+ и PD-L1- вариантов было 18,3 и 81,7\% (11 и 49 случаев) соответственно.

В группе больных с метастазами в лимфатических узлах (N1) данное соотношение равно 80,0 к 20,0\% (8 и 2 случая) соответственно ( $\mathrm{P}<0,001 ; \mathrm{PH}<0,001)$. Полученные данные говорят о наличии статистически достоверной зависимости между клеточной экспрессией PD-L1-рецепторов и метастазированием в лимфатические узлы, что имеет важное значение в оценке риска прогрессирования опухоли.

“При сравнении частоты отдалённого метастазирования было выявлено наличие достоверной зависимости этого показателя от статуса N" [7, с. 157; 9, с. 2293]. Так, в группе NO число больных с дистантными



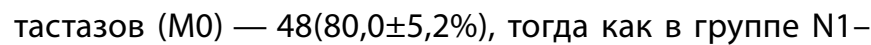
$6(60,0 \pm 15,5 \%)$ и $4(40,0 \pm 15,5 \%)$ соответственно; $\mathrm{P}=0,007$; $\mathrm{X} 2=7,179 ; \mathrm{PH}=0,008$. Структура частоты рецидивов при сравнении обеих групп такова: N0 - рецидивы развились у $10(16,7 \pm 4,8 \%)$, у 50(83,3 $44,8 \%)$ - рецидив отсут-


но, $\mathrm{P}=0,088 ; \mathrm{X}=2,917 ; \mathrm{PH}=0,090$. Статистический анализ подтверждает наличие корреляции между метастазированием в лимфатические узлы и отдалённой диссеминацией заболевания.

\section{Зак^ючение}

Проведённый анализ результатов иммуногистохимического окрашивания препаратов больных первично-операбельным РШМ выявил наличие определённых закономерностей между частотой регионарного метастазирования и PD-L1 и COX-2-статусом. Так, было установлено, что выраженность СОХ-2-экспрессии не влияет на частоту метастазов в лимфатические узлы $(\mathrm{P}=0,110, \mathrm{PH}=0,865)$, а также не коррелирует с клинической стадией PШM (PX2=0,010, PH=0,224). По PD-L1-статусу была выявлена достоверная зависимость с регионарным метастазированием - в 80,0\% случаях N1 опухоли были PD-L1-позитивны, тогда как при N0 PD-L1-позитивны были лишь 18,3\% новообразований $(\mathrm{P}<0,001 ; \mathrm{PH}<0,001)$. Также была установлена корреляция PD-L1-экспрессии с IIIC1 стадией заболевания $(\mathrm{P}<0,001, \mathrm{PH}<0,001)$. Было обнаружено, что наличие N1 соотносится с развитием отдалённых метастазов $60,0 \%$ против 20,0\% при NO ( $\mathrm{P}=0,007 ; \mathrm{PH}=0,008)$. Изучение частоты локальных рецидивов РШМ позволило установить отсутствие достоверной зависимости между данным показателем и статусом $\mathrm{N}(\mathrm{P}=0,088$; $\left.x^{2}=2,917\right)$.

Таким образом, у больных РШМ с метастазами в лимфатических узлах диагностика PD-L1-экспрессии имеет важное клиническое и прогностическое значение, иными словами, PD-L1-позитивность может быть расценена в качестве индикатора риска прогрессирования заболевания.

\section{ЛИТЕРАТУРА}

1. Bocanegra, A. and others. PD-L1 in systemic immunity uraveling its contribution to PD-1/PD-L1 blockade immunotherapy / A. Bocanegra, E. Blanco, H. Arasanz, L. Chocarro, M. Zuazo M., P. Morente, D. Escors, G. Kochan, G. Fernandez-Hinojal, R. Vera // International Journal of Molecular Sciences. — 2020. Vol. 21. Issue 16. — Pp. 1-17.

2. Chen Y. and others. Analysis of prognostic factors and clinical outcomes in uterine cervical carcinoma with isolated para-aortic lymph node recurrence. / Y. Chen, H. Lin, Ch. Wang // Am.J. Transl. Res. — 2019, — 11(12). — Pp. 7492-7502.

3. Chen Y. and others. Distribution patterns of lymph node metastasis in early stage invasive cervical cancer / Y. Chen, C. Fang, K. Zhang et al // — Baltimore: Medicine. - 2020, 0ct 16, - 99 (42). — Pp. 222-285.

4. Diggs, L.P. and others. Utility of PD-L1 immunohistochemystry assays for predicting PD-1/PD-L1 inhibitor response / L.P. Diggs, E.C. Hsueh // Biomarker Research. - 2017. Vol. 5. Issue 1. - Pp. 12. 
5. Duruisseaux, M. and others. Efficacy of PD1/PD-L1 immune checkpoint inhibitors and PD-L1 testing in thoracic cancers / M. Duruisseaux, I. Rouquette, J. Adam, A. Cortot, A. Cazes, L. Gibault, D. Damotte, S. Lantuejoul // Annales de Pathologie. — 2017. Vol. 37. Issue 1. — Pp. 61-78.

6. Heeren, M. and others. Prognostic effect of different PD-L1 expression patterns in squamous cell carcinoma and adenocarcinoma of the cervix / M. Heeren, S. Punt, M. Bleeker et al. // Mod Pathol. — 2016 Jul., — 29 (7). — Pp. 753-763.

7. Heskamp, S. and others. PD-L1 microspect imaging for longitudinal monitoring of PD-L1 expression in syngeneic and humanized mouse models for cancer / S. Heskamp, P.J. Wierstra, J.D.M. Molkenboer-Kuenen, G.W. Sandker, 0.C. Boerman, E.H.J.G. Aarntzen // Cancer Immunology Research. — 2019. Vol. 7. Issue 1. - Pp. 150-161

8. Liu, Z. and others. Patterns of lymph node metastasis in locally advanced cervical cancer / Z. Lui, K. Hu, A. Liu, J. Shen // — Baltimore: Medicine. - 2016 Sep., - 95 (39). - Pp. 148-154.

9. Li, T. and others. Exploring a tumor intrinsic PD-L1 signal with proximity dependent biotin identification in lung cancer cells / T. Li, F. Zhang, P. Qin, Y. Wang, A. Wang, L. Zhao, B. Xu, 0. Gao // Biochemistry. - 2019. Vol. 58. Issue 18. - Pp. 2293-2296.

10. Monneur, A. and others. PD-L1 expression and PD-1/PD-L1 inhibitors in reast cancer. / A. Monneur, A. Gonçalves, F. Bertucci // Bulletin du Cancer. — 2018. Vol. 105. Issue 3. - Pp. 263-274.

(c) Солтанова Ирада Фахраддин кызы ( geray.80@mail.ru ).

Журнал «Современная наука: актуальные проблемы теории и практики»

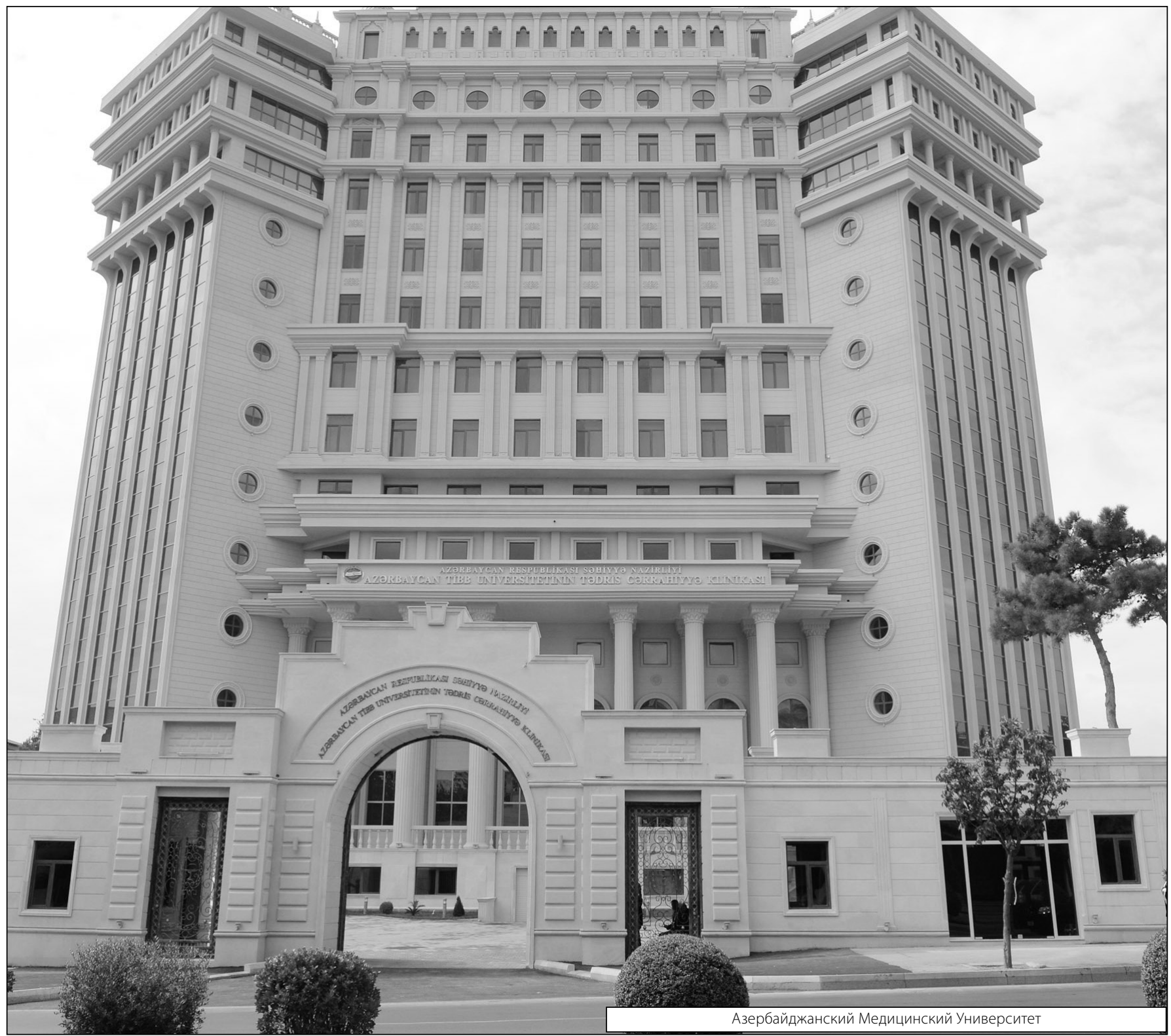

